\title{
An improved Pattern Search based algorithm to solve the Dynamic Economic Dispatch problem with valve-point effect
}

\author{
J.S. Alsumait ${ }^{\mathrm{a}, *}$, M. Qasem ${ }^{\mathrm{b}}$, J.K. Sykulski ${ }^{\mathrm{a}}$, A.K. Al-Othman ${ }^{\mathrm{c}}$ \\ ${ }^{a}$ Electrical Power Engineering Group, School of Electronics and Computer Science, University of Southampton, Southampton SO17 1BJ, United Kingdom \\ ${ }^{\mathrm{b}}$ Information: Signals, Images, Systems Group, School of Electronics and Computer Science, University of Southampton, Southampton SO17 1BJ, United Kingdom \\ ${ }^{\mathrm{c}}$ Electrical Engineering Department, College of Technological Studies, The Public Authority for Applied Education and Training, P.O. Box 33198, Alrawda 73452, Kuwait
}

\section{A R T I C L E I N F O}

\section{Article history:}

Received 7 July 2009

Accepted 18 February 2010

Available online 25 March 2010

\section{Keywords:}

Dynamic Economic Dispatch

Dynamic Economic and Emission Dispatch

Pattern Search

Valve-point effect

\begin{abstract}
A B S T R A C T
In this paper, an improved algorithm based on Pattern Search method (PS) to solve the Dynamic Economic Dispatch is proposed. The algorithm maintains the essential unit ramp rate constraint, along with all other necessary constraints, not only for the time horizon of operation $(24 \mathrm{~h})$, but it preserves these constraints through the transaction period to the next time horizon (next day) in order to avoid the discontinuity of the power system operation. The Dynamic Economic and Emission Dispatch problem (DEED) is also considered. The load balance constraints, operating limits, valve-point loading and network losses are included in the models of both DED and DEED. The numerical results clarify the significance of the improved algorithm and verify its performance.
\end{abstract}

(ㄷ) 2010 Elsevier Ltd. All rights reserved.

\section{Introduction}

The Dynamic Economic Dispatch (DED) formulation allows for a more advanced treatment of the Economic Dispatch problem (ED). The addition of certain periods of time in which the traditional ED is scheduled and operated, and the variation of the load demands over this period of time, have made the DED a more realistic representation of real conditions. The introduction of the ramp-up and ramp-down constraints in DED - in addition to the load balance constraints, operating limits, valve-point loading and network losses - has added an important aspect to the formulation. Furthermore, the concern about air pollution has also been taken into consideration. The combined Dynamic Economic and Emission Dispatch (DEED) have been addressed as a result of increased awareness of the need to reduce the release of harmful gases into atmosphere.

The DED was introduced in 1971 by Bechert and Kwanty [1]. The authors overcame the drawbacks of applying static optimization methods by combining economic load allocation and an additional control action. This combination was called the dynamic optimal control and is considered to be the foundation of the DED. Many modifications and additions have been proposed since to the original formulation. Ross and Kim [2] introduced a set of procedures and algorithms that protect the generation units from over-responding to the change of the predicted load. The authors split the large problem into smaller subproblems, and then solve

\footnotetext{
* Corresponding author. Tel.: +44 (0) 7767435 872; fax: +44 (0) 2380593709 .

E-mail address: jas2@soton.ac.uk (J.S. Alsumait).
}

each subproblem using forward dynamic programming. In [3] a particle swarm optimization (PSO) method is proposed to solve DED, in which the ramp rate limits, prohibited zones constraints and the non-smooth cost functions were all taken into consideration. A comparison between the proposed method and the genetic algorithm (GA) was undertaken to verify the quality of the algorithm. In the most recent publication [4], a new multiple tabu search algorithm (MTS) was presented and discussed. The authors considered most of the DED problem constraints, such as load demand, spinning reserve capacity, ramp rate limits and prohibited zones. The results of this novel algorithm were compared with PSO, ordinary tabu search, GA and simulated annealing (SA) methods to demonstrate the applicability and the superiority of MTS in DED applications.

The work reported in this paper regarding the DED problem has been conducted in the context of the following two journal papers. First, the authors of [5] used the simulated annealing (SA) method to solve the DED problem on a model that consists of five unit generators with non-smooth fuel cost functions. The unit ramp constraints for the five units were observed and maintained throughout the period of $24 \mathrm{~h}$ and the results were assumed to be global or near global. However, the authors admitted that the computing times were long and they suggested parallel processing as a solution. In the second paper, Basu [6] solved the Dynamic Economic Emission Dispatch (DEED) problem using evolutionary programming based fuzzy satisfying method. Moreover, the author treated the optimization problem as a minimax where the cost and the emission are competing to be the priority function by a decision maker (DM). Although the author listed the achieved optimal or near optimal total cost 
solutions, but unfortunately the outputs of each unit for the period of $24 \mathrm{~h}$ were not presented, as it was done in [5]. In addition, the solution of the EDE problem presented in [5] has not taken in consideration the consistency of the unit ramp constraints for all of the units in operation during the transaction time between one $24 \mathrm{~h}$ period to another. In other words, to avoid violating the unit ramp constrains after a period of $24 \mathrm{~h}$, the controller must shut down the whole power system and restart it again. This paper offers a solution how to rectify this drawback by improving the algorithm and making the necessary adjustments to ensure the continuity of the unit ramp constraint during the transaction time.

In this paper, a new approach to solve the Dynamic Economic Dispatch problem is developed and its results are compared with similar approaches in the literature. The proposed algorithm implements the Pattern Search (PS) method to solve the DED and DEED problems. After a brief introduction to PS is presented, the problem formulation is described in Section 3, followed in Section 4 by numerical results and comparison with other approaches $[5,6]$.

\section{Pattern Search method}

The Pattern Search (PS) optimization routine is an evolutionary technique that is suitable to solve a variety of optimization problems that lie outside the scope of the standard optimization methods. Generally, PS has the advantage of being very simple in concept, easy to implement and computationally efficient. Unlike other heuristic algorithms, such as genetic algorithms [7,8], PS possesses a flexible and well-balanced operator to enhance and adapt the global and fine tune local search. A useful review of direct search methods for unconstrained optimization is presented in [9], whereas details of the implementation adopted in this paper may be found in [10] and [11].

The Pattern Search (PS) algorithm proceeds by computing a sequence of points that may or may not approach the optimal value. The algorithm starts by establishing a set of points called a mesh, around the given point. This current point could be the initial starting point supplied by the user or it could be computed from the previous step of the algorithm. The mesh is formed by adding the current point to a scalar multiple of a set of vectors called a pattern. If a point in the mesh is found to improve the objective function at the current point, the new point becomes the current point at the next iteration. The flow chart of the process is shown in Fig. 1 (based on [10]).

\section{Problem formulation}

The formulation of the Dynamic Economic Dispatch consists of the traditional formulation of the ED scheduled over a period of time and supplemented by certain system bounds and operational constraints. In this section, the formulation of the DED is presented and the addition of an emission index is also considered. The objective function of the ordinary DED is as follows:

$f_{1}=F=\sum_{m=1}^{M} \sum_{i=1}^{N} F_{i m}\left(P_{i m}\right)$

with the incremental fuel cost functions of the generation units with valve-point loading represented as:

$F_{i m}\left(P_{i m}\right)=a_{i} P_{i m}^{2}+b_{i} P_{i m}+c_{i}+\left|e_{i} \sin \left(f_{i}\left(P_{i m i n}-P_{i m}\right)\right)\right|$

where $a_{i}, b_{i}, c_{i}, e_{i}, f_{i}$ are the cost coefficients of $i$ th unit, $P_{i m}$ is the output power of $i$ th unit at time $m, P_{i \min }$ is the lower generation bound for $i$ th unit, $N$ is the number of generation units, $M$ is the number of hours in the time horizon.

If the emission index is considered, then the following additional term should be added to the formulation

$f_{2}=E=\sum_{m=1}^{M} \sum_{i=1}^{N} E_{i m}\left(P_{i m}\right)$

and the amount of emission of each generator can be expressed $[6,12]$ by

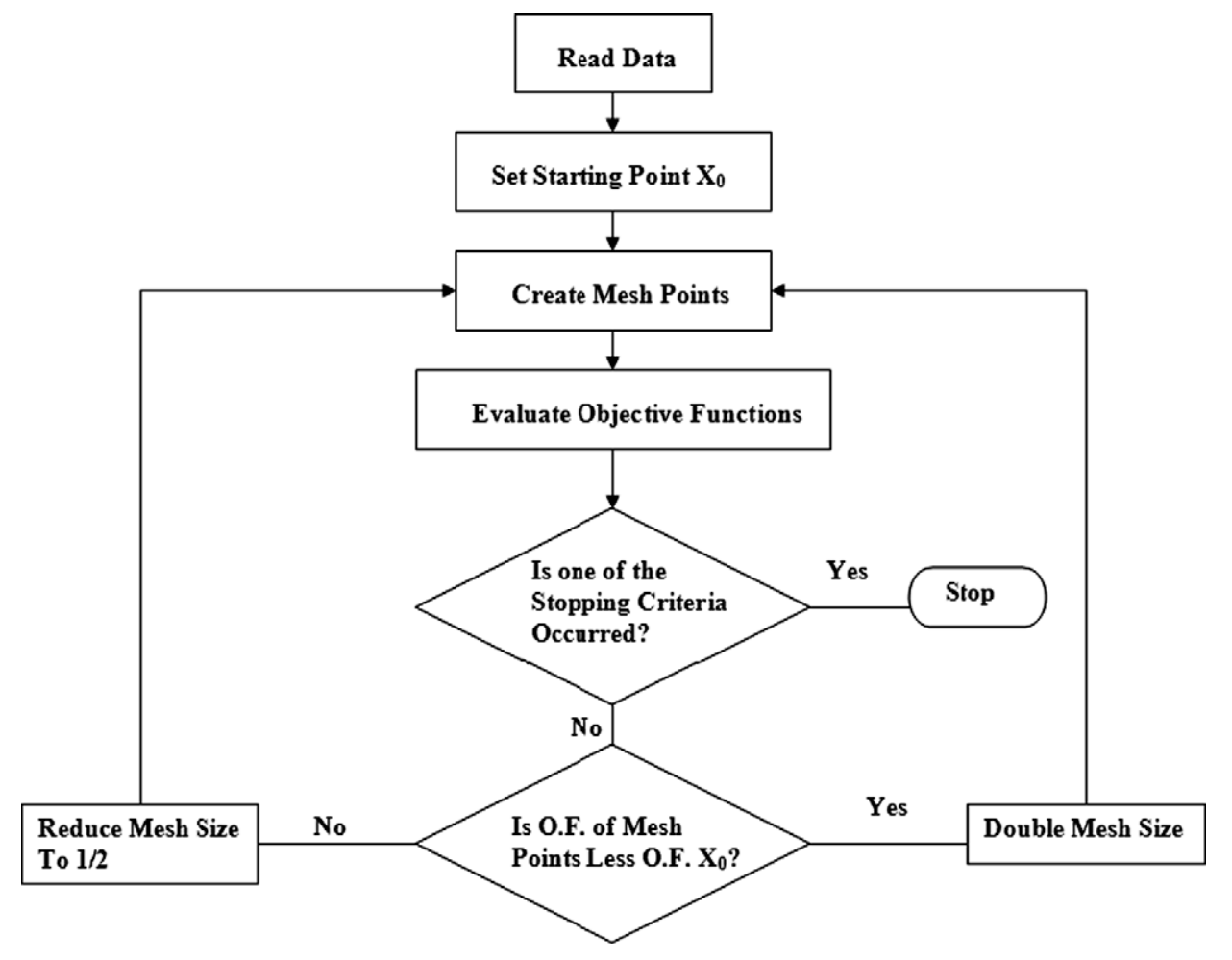

Fig. 1. PS flow chart [10]. 
$E_{i m}\left(p_{i m}\right)=\alpha_{i} P_{i m}^{2}+\beta_{i} P_{i m}+\gamma_{i}+\eta_{i} \exp \left(\delta_{i} P_{i m}\right)$

The following term is considered as the objective function to the combined economic and emission dispatch and the minimization problem is represented as

$\operatorname{Min}\left(f_{1}+f_{2}\right)=\operatorname{Min}(F+E)$

subject to

real power balance

$\sum_{i=1}^{N} P_{i m}=P_{D m}+P_{L m}$

real power operation limits

$P_{i \min }\left\langle P_{i m}\left\langle P_{i \max }, \quad i \in N, \quad m \in M\right.\right.$

generating unit ramp rate limits

$P_{i m}-P_{i(m-1)} \leqslant U R_{i}, \quad i \in N, \quad m \in M$

$P_{i(m-1)}-P_{\text {im }} \leqslant D R_{i}, \quad i \in N, \quad m \in M$

where $i=1,2, \ldots, T$. $P_{D m}$ is the load demand at time $m, P_{L m}$ is the transmission line losses at time $m, P_{i \max }$ is the upper generation bound for $i$ th unit, and $U R_{i}$ and $D R_{i}$ are the ramp-up and ramp-down rate limits of the $i$ th generator, respectively.

\subsection{System loss representation}

It should be noted that the so-called $B$-coefficients, or loss coefficients, have been adopted to model the system losses in the above formulations. The representation using $B$-coefficients is suitable for interpretation of the real power system losses under certain conditions. If the actual operating conditions are close to the base case, where $B$-constants were computed, then the $B$-coefficients method should compute the system losses with reasonably high accuracy $[13,14]$. In other words, the use of constant values for the loss coefficients in the equation for transmission losses yields good results when the coefficients are calculated for some average operating condition and assuming that extremely wide shifts of load between plants, or in the total load, do not occur In practice, large systems are often represented using just one set of loss coefficients which are sufficiently accurate throughout the daily variations of load on the system [14].

\section{Numerical results}

As mentioned before, the Dynamic Economic Dispatch (DED) formulation is a more realistic representation of a real scenario facing power station operation and control engineers, since DED solves the ED problem for $24 \mathrm{~h}$ with different load demands in each hour. However, the addition of new constraints in the DED formulation increases the complexity of the model compared to the ordinary ED. Consequently, the addition of the generation unit

Table 1

Data for five units system.

\begin{tabular}{llllll}
\hline Quantities & Unit 1 & Unit 2 & Unit 3 & Unit 4 & Unit 5 \\
\hline$a_{i}(\$ / \mathrm{h})$ & 25 & 60 & 100 & 120 & 40 \\
$b_{i}(\$ / \mathrm{MW} \mathrm{h})$ & 2.0 & 1.8 & 2.1 & 2.0 & 1.8 \\
$c_{i}\left(\$ /(\mathrm{MW})^{2} \mathrm{~h}\right)$ & 0.0080 & 0.0030 & 0.0012 & 0.0010 & 0.0015 \\
$e_{i}(\$ / \mathrm{h})$ & 100 & 140 & 160 & 180 & 200 \\
$f_{i}(1 / \mathrm{MW})$ & 0.042 & 0.040 & 0.038 & 0.037 & 0.035 \\
$P_{\text {imin }}(\mathrm{MW})$ & 10 & 20 & 30 & 40 & 50 \\
$P_{\text {imax }}(\mathrm{MW})$ & 75 & 125 & 175 & 250 & 300 \\
$U R(\mathrm{MW} / \mathrm{h})$ & 30 & 30 & 40 & 50 & 50 \\
$D R(\mathrm{MW} / \mathrm{h})$ & 30 & 30 & 40 & 50 & 50 \\
\hline
\end{tabular}

ramp rate limits, which restrict the change of the production of power in generating units between the hours of operation to a certain boundary, has increased the difficulty of the path to the optimal solution.

The section is divided into three sub-sections, the first describing the DED problem with fuel cost only, the second solving the DED problem with fuel cost and emission, and finally the third showing an improved algorithm to solve the second problem.

\subsection{Dynamic Economic Dispatch (fuel cost only)}

The system consists of five power generation units and only the fuel cost is considered in this case. The system data, transmission loss formula coefficients and load demand for $24 \mathrm{~h}$ were taken from [5] and are listed in Tables 1 and 2, respectively, whereas the transmission loss formula coefficients are:

$$
B=\left[\begin{array}{llllll}
0.000049 & 0.000014 & 0.000015 & 0.000015 & 0.000020 \\
0.000014 & 0.000045 & 0.000016 & 0.000020 & 0.000018 \\
0.000015 & 0.000016 & 0.000039 & 0.000010 & 0.000012 \\
0.000015 & 0.000020 & 0.000010 & 0.000040 & 0.000014 \\
0.000020 & 0.000018 & 0.000012 & 0.000014 & 0.000035
\end{array}\right] \text { per MW }
$$

In [5] a simulated annealing (SA) technique was used to solve this DED problem. The PS method has now been applied to the above system. A comparison between the two methods is given in Tables 3 and 4. It can be seen that the PS performance exceeds that of SA in terms of both fuel costs and execution times. Furthermore, both algorithms should maintain all constraints after the last hour when moving to hour number one on the next day; however, this is not the case. Hence the violated generators should be shut down and restarted again every day. To clarify this shortcoming, some of the cells in Table 4 were highlighted, namely units 2 and 5 which violated the generating unit ramp rate limits. To rectify this problem, an improved algorithm has been developed and will be presented in Sub-section 4.3 .

Detailed information regarding the system's power losses every hour of the day, total losses and the percentage of the total losses are given in Table 5. One of the main objectives of the proposed method is to reduce the generating power losses in the system, which will lead to minimizing the total cost. PS have decreased the total losses to $1.32 \%$ (192.2059 MW), which may be considered as an acceptable percentage in the electrical power industry.

\subsection{Dynamic Economic Dispatch (fuel and emission)}

In this case, the system consists of five power generation units and the system data, transmission loss formula coefficients, and load demand for $24 \mathrm{~h}$ were taken from [6]. The transmission loss formula coefficients and the load demand for $24 \mathrm{~h}$ are the same as in Sub-section 4.1. Furthermore, the data of the system is given in Table 6.

In this case, two approaches were attempted. The first technique solves the DED with a combined objective function. In other words, the fuel cost and the emission are combined into one objective function and then minimized. The second procedure solves the fuel cost and emission separately. It should be mentioned that no weighting factors were assigned to this minimization problem, and the algorithm's default weighting factor was 0.5 .

\subsubsection{Solving DED with combined objective functions}

The case of combining the cost and emission objective functions is presented in this section. Table 7 lists all the outputs of the five generators for the period of $24 \mathrm{~h}$. Moreover, the load demand and the unit ramp constraints are maintained throughout Table 7. 
Table 2

Load demand for $24 \mathrm{~h}$.

\begin{tabular}{|c|c|c|c|c|c|c|c|}
\hline Time (h) & Load (MW) & Time (h) & Load (MW) & Time (h) & Load (MW) & Time (h) & Load (MW) \\
\hline 1 & 410 & 7 & 626 & 13 & 704 & 19 & 654 \\
\hline 2 & 435 & 8 & 654 & 14 & 690 & 20 & 704 \\
\hline 3 & 475 & 9 & 690 & 15 & 654 & 21 & 680 \\
\hline 4 & 530 & 10 & 704 & 16 & 580 & 22 & 605 \\
\hline 5 & 558 & 11 & 720 & 17 & 558 & 23 & 527 \\
\hline 6 & 608 & 12 & 740 & 18 & 608 & 24 & 463 \\
\hline
\end{tabular}

Table 3

Comparison between SA and PS.

\begin{tabular}{lll}
\hline Method & SA & PS \\
\hline Best fuel cost (\$/day) & 47,356 & 46,530 \\
Best run time (s) & 351.98 & 272.2 \\
\hline
\end{tabular}

Table 4

Generators power production for $24 \mathrm{~h}$.

\begin{tabular}{ccccrl}
\hline Hour nos. & $P_{1}(\mathrm{MW})$ & $P_{2}(\mathrm{MW})$ & $P_{3}(\mathrm{MW})$ & $P_{4}(\mathrm{MW})$ & $P_{5}(\mathrm{MW})$ \\
\hline 1 & 24.906 & 21.200 & 75.570 & 77.970 & 214.07 \\
2 & 10.127 & 20.011 & 112.695 & 66.783 & 229.57 \\
3 & 10.000 & 20.000 & 112.673 & 107.675 & 229.52 \\
4 & 40.000 & 28.854 & 112.673 & 124.908 & 229.52 \\
5 & 57.127 & 40.350 & 112.673 & 124.908 & 229.52 \\
6 & 74.990 & 70.290 & 112.798 & 128.204 & 229.52 \\
7 & 72.974 & 90.794 & 112.798 & 128.204 & 229.52 \\
8 & 72.457 & 88.052 & 112.799 & 160.204 & 229.52 \\
9 & 49.623 & 98.539 & 112.673 & 209.815 & 229.52 \\
10 & 64.011 & 98.540 & 112.673 & 209.815 & 229.52 \\
11 & 48.365 & 98.540 & 144.674 & 209.816 & 229.52 \\
12 & 68.948 & 98.540 & 144.674 & 209.816 & 229.52 \\
13 & 63.819 & 98.540 & 144.67 & 177.816 & 229.52 \\
14 & 65.578 & 114.54 & 112.67 & 177.816 & 229.52 \\
15 & 60.739 & 114.54 & 80.67 & 177.816 & 229.52 \\
16 & 30.739 & 86.402 & 112.673 & 127.816 & 229.52 \\
17 & 40.238 & 86.402 & 112.673 & 95.816 & 229.52 \\
18 & 50.207 & 98.540 & 112.673 & 124.91 & 229.52 \\
19 & 75.000 & 98.540 & 112.673 & 147.32 & 229.52 \\
20 & 75.000 & 100.02 & 112.673 & 197.32 & 229.52 \\
21 & 75.000 & 98.540 & 112.673 & 174.06 & 229.52 \\
22 & 60.784 & 82.540 & 145.673 & 126.06 & 197.52 \\
23 & 36.140 & 75.376 & 113.673 & 142.06 & 165.52 \\
24 & 50.871 & 91.377 & 81.673 & 110.06 & 133.52 \\
\hline
\end{tabular}

Table 5

Power losses for $24 \mathrm{~h}$.

\begin{tabular}{cclc}
\hline Hour nos. & Power losses $(\mathrm{MW})$ & Hour nos. & Power losses $(\mathrm{MW})$ \\
\hline 1 & 3.717 & 13 & 10.366 \\
2 & 4.186 & 14 & 10.127 \\
3 & 4.868 & 15 & 9.288 \\
4 & 5.955 & 16 & 7.150 \\
5 & 6.578 & 17 & 6.649 \\
6 & 7.803 & 18 & 7.847 \\
7 & 8.290 & 19 & 9.049 \\
8 & 9.032 & 20 & 10.53 \\
9 & 10.168 & 21 & 9.791 \\
10 & 10.559 & 22 & 7.575 \\
11 & 10.914 & 23 & 5.767 \\
12 & 11.497 & 24 & 4.499 \\
Total losses $=192.21(\mathrm{MW})$ & Percentage of losses $=1.32 \%$ \\
\hline
\end{tabular}

The total fuel cost and the emission index produced by PS are $\$ 47,911$ per day and $18,927 \mathrm{lb} /$ day, respectively. From Figs. 2
Table 6

System data.

\begin{tabular}{llllll}
\hline Quantities & Unit 1 & Unit 2 & Unit 3 & Unit 4 & Unit 5 \\
\hline$a(\$ / \mathrm{h})$ & 25 & 60 & 100 & 120 & 40 \\
$b(\$ / \mathrm{MW} \mathrm{h})$ & 2.0 & 1.8 & 2.1 & 2.0 & 1.8 \\
$\left.c(\$ / \mathrm{MW})^{2} \mathrm{~h}\right)$ & 0.0080 & 0.0030 & 0.0012 & 0.0010 & 0.0015 \\
$d(\$ / \mathrm{h})$ & 100 & 140 & 160 & 180 & 200 \\
$e(\mathrm{rad} / \mathrm{MW})$ & 0.042 & 0.040 & 0.038 & 0.037 & 0.035 \\
$\alpha(l b / \mathrm{h})$ & 80 & 50 & 60 & 45 & 30 \\
$\beta(l b / \mathrm{MW})$ & -0.805 & -0.555 & -1.355 & -0.600 & -0.555 \\
$\gamma\left(l b / \mathrm{MW}^{2} \mathrm{~h}\right)$ & 0.0180 & 0.0150 & 0.0105 & 0.0080 & 0.0120 \\
$\eta(l b / \mathrm{h})$ & 0.6550 & 0.5773 & 0.4968 & 0.4860 & 0.5035 \\
$\delta(l b / \mathrm{MW})$ & 0.02846 & 0.02446 & 0.02270 & 0.01948 & 0.02075 \\
$P_{\text {imin }}(\mathrm{MW})$ & 10 & 20 & 30 & 40 & 50 \\
$P_{\text {imax }}(\mathrm{MW})$ & 75 & 125 & 175 & 250 & 300 \\
$U R(\mathrm{MW} / \mathrm{h})$ & 30 & 30 & 40 & 50 & 50 \\
$D R(\mathrm{MW} / \mathrm{h})$ & 30 & 30 & 40 & 50 & 50 \\
\hline
\end{tabular}

Table 7

Generators power production for $24 \mathrm{~h}$.

\begin{tabular}{cccccc}
\hline Hour nos. & $P_{1}(\mathrm{MW})$ & $P_{2}(\mathrm{MW})$ & $P_{3}(\mathrm{MW})$ & $P_{4}(\mathrm{MW})$ & $P_{5}(\mathrm{MW})$ \\
\hline 1 & 16.927 & 86.655 & 59.803 & 180.54 & 69.929 \\
2 & 46.927 & 98.540 & 34.315 & 209.81 & 50.000 \\
3 & 75.000 & 98.540 & 46.925 & 209.81 & 50.000 \\
4 & 75.000 & 98.540 & 86.925 & 209.81 & 65.937 \\
5 & 75.000 & 98.540 & 112.67 & 209.81 & 68.732 \\
6 & 75.000 & 98.540 & 113.78 & 209.81 & 118.73 \\
7 & 73.574 & 98.540 & 112.66 & 209.81 & 139.73 \\
8 & 70.320 & 98.540 & 112.66 & 209.81 & 171.73 \\
9 & 74.206 & 98.540 & 145.66 & 209.81 & 171.73 \\
10 & 74.961 & 104.40 & 174.97 & 209.81 & 150.17 \\
11 & 74.923 & 98.556 & 174.98 & 209.81 & 172.48 \\
12 & 63.544 & 98.556 & 174.98 & 209.81 & 204.48 \\
13 & 58.467 & 98.556 & 174.98 & 209.81 & 172.48 \\
14 & 60.132 & 114.56 & 174.98 & 209.81 & 140.48 \\
15 & 55.194 & 114.56 & 142.98 & 209.81 & 140.48 \\
16 & 43.254 & 98.558 & 110.98 & 177.81 & 156.48 \\
17 & 52.662 & 98.558 & 110.98 & 145.81 & 156.48 \\
18 & 71.799 & 98.558 & 142.98 & 145.81 & 156.48 \\
19 & 75.000 & 98.540 & 175.00 & 174.54 & 139.75 \\
20 & 75.000 & 114.78 & 175.00 & 209.81 & 139.76 \\
21 & 75.000 & 98.540 & 166.52 & 209.81 & 139.76 \\
22 & 60.946 & 82.540 & 135.52 & 177.81 & 155.76 \\
23 & 53.984 & 98.540 & 112.67 & 127.81 & 139.76 \\
24 & 36.844 & 98.540 & 80.673 & 159.81 & 91.760 \\
\hline & & & & & \\
& & & &
\end{tabular}

and 3, it can be easily seen that PS yields the lowest overall fuel cost and the lowest emission index compared to EP and SA.

Table 8 shows the losses of the five generators every hour of the $24 \mathrm{~h}$ period of operation. In addition, the total losses of the system and the percentage of these losses to the total generation of the units are also presented. It is worth mentioning that the default weighting factor of 0.5 was used in this part of the paper.

\subsubsection{Solving DED with separated objective functions}

In this sub-section the DEED problem was solved twice with each objective function separately and results are shown in Table 9. 
Fuel Cost \$/day

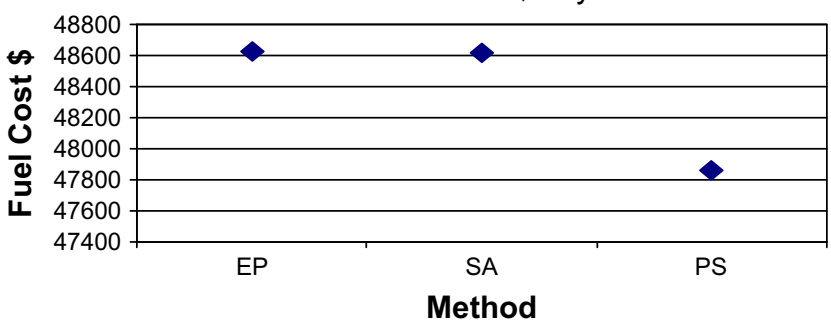

Fig. 2. Fuel cost comparison.

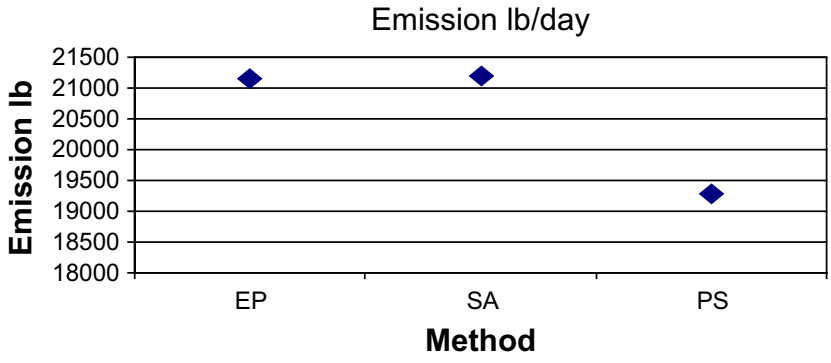

Fig. 3. Emission comparison.

The PS performed better with the combined objective function (Section 4.2.1) than this trial. Further trials were carried out but no improvement in the emission index was achieved.

\subsection{Dynamic Economic Dispatch (improved algorithm)}

An improved version of the algorithm which can eliminate the drawback of violating the unit ramp constraint after the $24 \mathrm{~h}$ period is presented here. It guarantees the consistency of the unit ramp rate for the next day of the units operation (which the previous method in [5] did not). Due to the significance of the unit ramp rate constraint in electric power systems, improvements to the initial program code were undertaken to resolve the weakness that

Table 8

Power losses for $24 \mathrm{~h}$

\begin{tabular}{cclc}
\hline Hour nos. & Power losses $(\mathrm{MW})$ & Hour nos. & Power losses $(\mathrm{MW})$ \\
\hline 1 & 3.856 & 13 & 10.30 \\
2 & 4.598 & 14 & 9.970 \\
3 & 5.280 & 15 & 9.032 \\
4 & 6.217 & 16 & 7.092 \\
5 & 6.761 & 17 & 6.501 \\
6 & 7.869 & 18 & 7.638 \\
7 & 8.317 & 19 & 8.838 \\
8 & 9.062 & 20 & 10.36 \\
9 & 9.949 & 21 & 9.638 \\
10 & 10.32 & 22 & 7.584 \\
11 & 10.76 & 23 & 5.773 \\
12 & 11.38 & 24 & 4.632 \\
Total losses $=191.73(\mathrm{MW})$ & Percentage of losses $=1.32 \%$ \\
\hline
\end{tabular}

Table 9

Results of separated objective functions.

\begin{tabular}{lll}
\hline & EP & PS \\
\hline Fuel cost (\$/day) & 46,777 & 46,530 \\
Emission (lb/day) & 17,966 & 18,192 \\
Run time & - & 294.94 \\
\hline
\end{tabular}

Table 10

Generators power production for $24 \mathrm{~h}$

\begin{tabular}{cccccc}
\hline Hour nos. & $P_{1}(\mathrm{MW})$ & $P_{2}(\mathrm{MW})$ & $P_{3}(\mathrm{MW})$ & $P_{4}(\mathrm{MW})$ & $P_{5}(\mathrm{MW})$ \\
\hline 1 & 16.8272 & 36.051 & 107.190 & 102.65 & 150.75 \\
2 & 41.5366 & 20.000 & 112.673 & 124.91 & 139.76 \\
3 & 58.7964 & 43.480 & 112.673 & 124.91 & 139.76 \\
4 & 75.0000 & 73.480 & 122.627 & 124.91 & 139.76 \\
5 & 75.0000 & 98.540 & 126.239 & 124.91 & 139.76 \\
6 & 75.0000 & 98.540 & 166.239 & 136.08 & 139.76 \\
7 & 75.0000 & 98.540 & 175.000 & 145.78 & 139.76 \\
8 & 75.0000 & 98.540 & 175.000 & 174.54 & 139.76 \\
9 & 75.0000 & 100.34 & 175.000 & 209.82 & 139.76 \\
10 & 75.0000 & 114.78 & 175.000 & 209.82 & 139.76 \\
11 & 75.0000 & 125.00 & 175.000 & 209.82 & 146.04 \\
12 & 75.0000 & 125.00 & 175.000 & 209.82 & 166.63 \\
13 & 75.0000 & 114.78 & 175.000 & 209.82 & 139.76 \\
14 & 75.0000 & 100.23 & 175.000 & 209.92 & 139.76 \\
15 & 75.0000 & 102.50 & 135.893 & 209.83 & 139.77 \\
16 & 62.0234 & 86.499 & 168.89 & 177.83 & 91.77 \\
17 & 46.0234 & 88.296 & 168.89 & 177.83 & 83.51 \\
18 & 16.0234 & 98.540 & 157.98 & 209.82 & 133.51 \\
19 & 39.8480 & 98.540 & 175.00 & 209.82 & 139.76 \\
20 & 69.8480 & 119.96 & 175.00 & 209.82 & 139.76 \\
21 & 46.2456 & 119.96 & 142.00 & 209.82 & 171.76 \\
22 & 33.2429 & 103.95 & 110.00 & 177.82 & 187.76 \\
23 & 33.50 & 87.95 & 78.00 & 193.81 & 139.76 \\
24 & 12.52 & 65.80 & 105.50 & 143.90 & 139.76 \\
\hline
\end{tabular}

Table 11

Comparison between EP, SA, and PS results.

\begin{tabular}{llll}
\hline & EP & SA & PS \\
\hline Fuel cost (\$/day) & 48,628 & 48,621 & 47,911 \\
Emission (lb/day) & 21,154 & 21,188 & 18,927 \\
Run time (s) & - & - & 514.25 \\
\hline
\end{tabular}

appeared in the previous algorithms. The implementation of this improved version of the algorithm has been carried on the combined Dynamic Economic emission fuel (DEED) problem mentioned in Sub-section 4.2.1.

The improvements that were incorporated into the original algorithm may be summarized as follows: (1) the interconnection between the last hour and the next first hour in the time horizons, (2) the redefinition of the upper and lower limits after the addition of the unit's ramp-up and ramp-down constrains. The first part of the improvements has eliminated the violation of the unit ramp rate constraints between the last hour of the operation period and the first hour in the next operation period. However, this has led to the increase of the computation time, and this has instigated the second feature. Since the DED has unit ramp rate constraints, when the search starts, new upper and lower limits are defined in accordance with the units' ramp-up and ramp-down limits. As a result, the improved algorithm does not need to search the whole range between the upper and lower limits of the generator, and the redefined range is set by the units' ramp-up and ramp-down bounds. This feature has reduced the scope of search leading to decreased computational times.

Table 10 illustrates the outcome for the five units over the $24 \mathrm{~h}$ operation time. It can be seen that the unit ramp rate constraint has not been violated for the five generators. In other words, the units can be operated after the 24th hour to the 1 st in the next day without the need to be concerned about the unit ramp rate limits of the units. This has not been the case in the method proposed in [5], where there are three unit ramp rate violations in units 2, 3 and 5 (see Table 3 in [5]).

Table 11 shows the comparison between the proposed method and the results of EP and SA that have been reported in [6]. The PS 
has achieved the lowest fuel cost and emission when compared to EP and SA. However, the computation time of the algorithm increased to $514.25 \mathrm{~s}$.

\section{Conclusion}

In this paper, an improved algorithm based on the Pattern Search method has been employed successfully to solve the Dynamic Economic Dispatch and Dynamic Economic and Emission Dispatch problems. The proposed algorithm maintains the units' ramp-up and ramp-down constraints after the end of the time horizon of operation to ensure the cyclic continuity. This feature has not been previously considered in the literature. Many other conditions and constrains were also observed and sustained such as load balance, operation limits, valve-point loading, and network losses. The proposed algorithm has been demonstrated to perform well when applied to the DED and DEED problems.

\section{References}

[1] Bechert TE, Kwanty HG. On the optimal dynamic dispatch of real power. IEEE Trans Power Ap Syst 1972;PAS-91:889-98.

[2] Ross DW, Kim S. Dynamic economic dispatch of generation. IEEE Trans Power Ap Syst 1980;PAS-99:2060-8.
[3] Zwe-Lee G. Particle swarm optimization to solving the economic dispatch considering the generator constraints. IEEE Trans Power Syst 2003;18: 1187-95.

[4] Pothiya S, Ngamroo I, Kongprawechnon W. Application of multiple tabu search algorithm to solve dynamic economic dispatch considering generator constraints. Energy Convers Manage 2008;49:506-16.

[5] Panigrahi CK, Chattopadhyay PK, Chakrabarti RN, Basu M. Simulated annealing technique for dynamic economic dispatch. Electric Power Compo Syst 2006;34:577-86.

[6] Basu M. Dynamic economic emission dispatch using evolutionary programming and fuzzy satisfying method. Int J Emerg Electric Power Syst 2007;8:1-15.

[7] Goldberg DE. Genetic algorithms in search, optimization, and machine learning. Reading, Harlow, Mass.: Addison-Wesley; 1989.

[8] Michalewicz Z. Genetic algorithms + data structures = evolution programs. 3rd rev. and extended ed. Berlin, New York: Springer-Verlag; 1996.

[9] Lewis RM, Torczon V, Trosset MW. Direct search methods: then and now. J Comput Appl Math 2000;124:191-207.

[10] Al-Sumait JS, Al-Othman AK, Sykulski JK. Application of pattern search method to power system valve-point economic load dispatch. Int J Electric Power Energy Syst 2007;29:720-30.

[11] Al-Sumait JS, Sykulski JK, Al-Othman AK. Solution of different types of economic load dispatch problems using a pattern search method. Electric Power Compo Syst 2008;36:250-65.

[12] Gent MR, Lamont JW. Minimum-emission dispatch. IEEE Trans Power Ap Syst 1971;PAS-90:2650-60.

[13] Saadat H. Power system analysis. Boston, Mass.; London: WCB/McGraw-Hill; 1999.

[14] Grainger JJ, Stevenson WD. Power system analysis. New York: McGraw-Hill; 1994. 